\title{
Indicadores de especialização produtiva agropecuária para caracterização de municípios do estado de São Paulo (Brasil)
}

\section{Indicators of agricultural productive specialization to characterize municipalities in the state of São Paulo (Brazil)}

\section{Paulo André de Oliveira}

Centro Estadual de Educação Tecnológica Paula Souza

Faculdade de Tecnologia de Botucatu (FATEC-BT)

paulo.oliveira108@fatec.sp.gov.br

\section{Sergio Augusto Rodrigues}

Universidade Estadual Paulista "Júlio de Mesquita Filho" (UNESP) - Faculdade de Ciências Agronômicas (FCA) Campus Botucatu SP - Departamento de Bioprocessos e Biotecnologia (DBB)

sergio.rodrigues@unesp.br

\section{Carlos Roberto Padovani}

Universidade Estadual Paulista "Júlio de Mesquita Filho" (UNESP) - Instituto de Biociências (IBB), Campus Botucatu-SP - Departamento de Bioestatística

cr.padovani@unesp.br

\section{Ricardo Ghantous Cervi}

Universidade Estadual Paulista "Júlio de Mesquita Filho" (UNESP - Câmpus Experimental de Itapeva ricardo.cervi@unesp.br 


\section{Resumo}

A hipótese geral desta pesquisa foi que a produção agropecuária se apresenta de forma desigual no território com expressivas diferenças de produtividade e rendimento financeiro em conjunto de produtos. O objetivo deste trabalho foi permitir um conhecimento mais abrangente das características de especialização e concentração da produção agropecuária, com base em conjuntos de produtos agropecuários ponderado pela sua área ocupada. As informações de 621 municípios paulistas com produção agropecuária constituíram um vetor aleatório, denominado de contribuição financeira por hectare, formado por variáveis de produção e área produtiva total dos municípios. Por meio de três indicadores analisaram a concentração produtiva municipal. O grau de concentração produtiva por conjunto de produtos demonstrou que a maior concentração produtiva foi acompanhada por maior rentabilidade por hectare. O forte dilema entre diversificação para diluir os riscos de mercado e a especialização para aumentar a eficiência produtiva foi verificado em todos os conjuntos de produtos.

Palavras-chaves: diversificação, produtividade, especialização

\section{Abstract}

The general hypothesis of this research was that agricultural production presents itself unevenly in the territory with significant differences in productivity and financial income in terms of products. The objective of this work was to allow a more comprehensive knowledge of the characteristics of specialization and concentration of agricultural production based on sets of agricultural products weighted by their occupied area. The information from 621 São Paulo municipalities with agricultural production constituted a random vector, called financial contribution per hectare, formed by production variables and the total productive area of the municipalities. Using three indicators, they analyzed the municipal productive concentration. The degree of productive concentration per set of products demonstrated that the highest productive concentration was accompanied by greater profitability per hectare. The strong dilemma between diversification to dilute market risks and specialization to increase production efficiency was seen in all product sets.

Keywords: diversification, productivity, specialization

Journal of Economic Literature (JEL): R1, R14, O18 


\section{Introdução}

O desenvolvimento econômico é constantemente buscado nas ações de políticas públicas, porque se espera que este contribua para a melhoria na qualidade de vida das pessoas de uma região, a qual pode ser definida como um município ou até mesmo um país. Nesse sentido, ocorre uma intensificação de esforços para apontar caminhos para a definição de estratégias que venham a fortalecer o desenvolvimento rural no Brasil. Destacam-se as linhas de pesquisa que tratam de questões como o redimensionamento dos espaços rurais (Veiga et al., 2001), o aumento de importância de formas não agrícolas de ocupação econômica dos espaços rurais (Silva e Grossi, 2001) e, ainda, abordagens institucionalistas (Abramovay, 2003). A questão que se observa é que existem diferentes estágios de desenvolvimento e de crescimento econômico em uma mesma região.

As disparidades regionais estão presentes no Brasil desde o início de sua história, ocasionadas por diferentes fatores, entre eles políticos e econômicos. Essas disparidades afetam o país não somente no contexto estadual, mas também em um contexto municipal, fazendo com que seja perceptível a emergência de diferentes níveis de desenvolvimento em cidades de um mesmo estado e até mesmo de uma mesma microrregião (Medeiros Costa, Ferreira, Braga e Abrantes, 2012).

De acordo com Menezes e Azzoni (2006), as desigualdades regionais ocorrem tanto por fatores relacionados à oferta de trabalho como por fatores de demanda por trabalho. Os enfoques gerais ao tratamento das desigualdades regionais se diferenciam por enfatizarem mais ou menos esses dois conjuntos de fatores. Esses enfoques podem ser baseados no capital humano e de crescimento (Ferreira e Ellery Junior, 1996) e nos específicos da economia regional (Combes, Duranton e Cobillon, 2008), que também destacam a importância de atributos locais.

Uma política de desenvolvimento regional, muitas vezes para uma macrorregião ou até mesmo um estado, é muito ampla e desigual. O processo de desenvolvimento econômico e social não ocorre de maneira igual e simultânea em todos os locais. Esse processo ocorre de forma irregular e, uma vez iniciado em determinados pontos, fortalece áreas mais dinâmicas que apresentam maior potencial de desenvolvimento (Lima e Simões, 2010).

Segundo os cálculos de Shankar e Shah (2003), os países em desenvolvimento são, em média, seis vezes mais desiguais do que os países desenvolvidos em termos regionais. Os autores argumentam que dois fatores podem conduzir a essa si- 
tuação: no primeiro, os países em desenvolvimento se preocupam mais com o crescimento e, devido à escassez de recursos, tendem a concentrá-los nas regiões mais desenvolvidas; no segundo, os países em desenvolvimento possuem mercados com mais imperfeições, o que leva ao aumento de barreiras inter-regionais, dificultando a mobilidade dos fatores produtivos e da produção.

Belik (2015) destaca que as desigualdades em termos de tamanho da área, aporte tecnológico, gestão e utilização da força de trabalho são enormes quando comparamos as diferentes regiões e países. Mesmo entre os agricultores familiares, as diferenças são significativas. Nesse contexto, segundo Souza, Ney, Fornazier e Ponciano (2015), podem ser encontrados desde produtores plenamente inseridos no mercado até unidades que produzem praticamente para a subsistência, em alguns casos padecendo, inclusive, de insegurança alimentar.

Para Gasques, Bastos, Valdes, e Bacchi (2013) os reflexos do aumento da produtividade no Brasil podem ser observados nas taxas negativas do uso de insumos totais na última década. As áreas de pastagem tiveram reduções significativas, enquanto o efetivo de animais teve grande aumento. Também se observa uma redução da mão de obra na agricultura e um padrão contínuo e intenso de crescimento no índice do capital, resultando na implementação da modernização agrícola.

Segundo Silva e Ferreira (2016) a produtividade da mão de obra foi o principal componente associado ao acréscimo da produtividade total dos fatores (PTF). No período de 1975 a 2011, a estimativa da taxa anual de crescimento da produtividade da mão de obra foi superior à da produtividade da terra $-4,46 \%$ contra $3,81 \%$. 0 efeito da produtividade da terra sobre a PTF também foi expressivo nesse período $(284,21 \%)$, mostrando-se superior à produtividade do capital (207,22\%).

O estímulo ao desenvolvimento econômico de uma região necessita de uma base de critérios de análise para se implementar ações que visem a melhoria do aparelho produtivo e como consequência o padrão de vida das pessoas daquela região. Uma estratégia de desenvolvimento que busque reduzir as desigualdades territoriais precisa estimular as atividades econômicas de uma região (Silva e Andraz, 2004).

No conjunto dos métodos de análise regional Haddad (1989) destacou que se encontram as medidas de localização e especialização, que servem para identificar padrões de comportamento dos setores produtivos no espaço econômico, bem como padrões diferenciais de estruturas produtivas entre as várias regiões. No entanto, são de natureza descritiva e de escopo um tanto quanto limitado, embora se justifiquem em estudos de caráter exploratório. Segundo Araújo (2013), o avanço nos estudos da economia regional e nas suas técnicas de análise permitiu uma observação mais profunda do processo de desenvolvimento de um espaço territorial e contribuem para fortalecer esse processo.

As aplicações empíricas da concentração se suportam em medidas de concentração. Essas medidas têm o objetivo de captar de que forma agentes econô 
tuação: no primeiro, os países em desenvolvimento se preocupam mais com o crescimento e, devido à escassez de recursos, tendem a concentrá-los nas regiões mais desenvolvidas; no segundo, os países em desenvolvimento possuem mercados com mais imperfeições, o que leva ao aumento de barreiras inter-regionais, dificultando a mobilidade dos fatores produtivos e da produção.

Belik (2015) destaca que as desigualdades em termos de tamanho da área, aporte tecnológico, gestão e utilização da força de trabalho são enormes quando comparamos as diferentes regiões e países. Mesmo entre os agricultores familiares, as diferenças são significativas. Nesse contexto, segundo Souza, Ney, Fornazier e Ponciano (2015), podem ser encontrados desde produtores plenamente inseridos no mercado até unidades que produzem praticamente para a subsistência, em alguns casos padecendo, inclusive, de insegurança alimentar.

Para Gasques, Bastos, Valdes, e Bacchi (2013) os reflexos do aumento da produtividade no Brasil podem ser observados nas taxas negativas do uso de insumos totais na última década. As áreas de pastagem tiveram reduções significativas, enquanto o efetivo de animais teve grande aumento. Também se observa uma redução da mão de obra na agricultura e um padrão contínuo e intenso de crescimento no índice do capital, resultando na implementação da modernização agrícola.

Segundo Silva e Ferreira (2016) a produtividade da mão de obra foi o principal componente associado ao acréscimo da produtividade total dos fatores (PTF). No período de 1975 a 2011, a estimativa da taxa anual de crescimento da produtividade da mão de obra foi superior à da produtividade da terra $-4,46 \%$ contra 3,81 \%. 0 efeito da produtividade da terra sobre a PTF também foi expressivo nesse período (284,21\%), mostrando-se superior à produtividade do capital (207,22 \%).

O estímulo ao desenvolvimento econômico de uma região necessita de uma base de critérios de análise para se implementar ações que visem a melhoria do aparelho produtivo e como consequência o padrão de vida das pessoas daquela região. Uma estratégia de desenvolvimento que busque reduzir as desigualdades territoriais precisa estimular as atividades econômicas de uma região (Silva e Andraz, 2004).

No conjunto dos métodos de análise regional Haddad (1989) destacou que se encontram as medidas de localização e especialização, que servem para identificar padrões de comportamento dos setores produtivos no espaço econômico, bem como padrões diferenciais de estruturas produtivas entre as várias regiões. No entanto, são de natureza descritiva e de escopo um tanto quanto limitado, embora se justifiquem em estudos de caráter exploratório. Segundo Araújo (2013), o avanço nos estudos da economia regional e nas suas técnicas de análise permitiu uma observação mais profunda do processo de desenvolvimento de um espaço territorial e contribuem para fortalecer esse processo.

As aplicações empíricas da concentração se suportam em medidas de concentração. Essas medidas têm o objetivo de captar de que forma agentes econô- 
micos evidenciam um "comportamento dominante" em determinado mercado, e nesse sentido os diferentes indicadores consideram as participações no mercado dos agentes, por exemplo, por meio do número de empregados de cada empresa no total dos vínculos empregatícios do setor, de acordo com diferentes critérios de ponderação.

Medidas de concentração industrial são úteis para indicar, preliminarmente, os setores para os quais se espera que o "poder de mercado" seja significativo (Resende e Boff, 2013; Liu, Mirzaei e Vandoros, 2014). Além disso, os índices de concentração são utilizados para demonstrar de maneira sintética um indicador de concorrência de um mercado específico, onde quanto maior a concentração menor é o grau da concorrência (Setiawan, Emvalomatis e Lansink, 2012; Resende e Boff, 2013). Para Resende (1994), existem duas categorias de indicadores de concentração, a saber: parciais ou sumárias, podendo ser estas positivas ou normativas, onde cada uma delas está atrelada a um ou mais índices. Na primeira categoria ("parciais"), - na qual se encontra o índice "Razão de Concentração" - verifica-se a utilização de apenas parte do mercado, usualmente os maiores do segmento em análise. Para a mensuração deste índice basta utilizar algumas informações do setor (não sendo necessário, por exemplo, o número total de firmas). Já na segunda categoria ("sumária") existe a necessidade de se utilizar informações de todas as firmas do setor que se quer fazer a verificação. Exemplos desses índices são "Hirschman-Herfindahl" (HHI) e o "Coeficiente de Entropia de Theil" (ET).

O estado de São Paulo desenvolveu uma agricultura de áreas altamente especializadas, porém muitas delas apresentaram especialização em certas atividades agrícolas, mas não conseguiram superar dificuldades, algumas ligadas às restrições naturais que condicionaram seu desenvolvimento agrícola e social. No âmbito do desenvolvimento regional as diferenças de produtividade, não são detectadas claramente com a divisão político-administrativa constituída por áreas adjacentes de municípios, como ocorre nas regiões dos Escritórios de Desenvolvimento Rural do Estado de São Paulo, as quais muitas vezes não apresentam desagregação ou agregação espacial adequada para a execução de políticas públicas devido à heterogeneidade existente entre os municípios.

A hipótese geral desta pesquisa foi que a produção agropecuária se realiza de forma desigual no território com expressivas diferenças de produtividade e rendimento financeiro em conjunto de produtos que acabam por determinar a característica produtiva de um município ou região. Contribuições mais recentes demonstram que outros mecanismos de convergência (de modernização, de renda, entre outros) ajudam a especificar melhor a natureza da desigualdade espacial do desenvolvimento.

A literatura econômica apresenta diversas estratégias para o desenvolvimento de uma região ou país e como explicou Aldeman (2002) o que é bom em um estágio do desenvolvimento pode ser ruim para o seguinte. Neste sentido, a contribuição 
deste trabalho se limita em uma metodologia para mensurar especialização produtiva da agropecuária paulista, sem intenção de debater estratégias de desenvolvimento econômico e social.

O objetivo deste trabalho foi conhecer de forma mais abrangente as características de especialização e concentração da produção agropecuária, com base em conjuntos de produtos agropecuários ponderado pela sua área ocupada. Desta forma, se fornece base para um aprofundamento em determinado setor de interesse.

\section{Metodologia}

Foram levantadas informações relativas aos municípios paulistas com produção agropecuária e uso da área rural no ano de 2008, totalizando 621 municípios. $O$ ano de 2008 foi considerado em razão da disponibilidade oficial de informações coincidentes para as variáveis utilizadas neste estudo.

Tabela 1. Descrição das variáveis de valor bruto de produção agropecuária (em reais) e uso da área rural (ha) avaliadas nos municípios do estado de São Paulo (Brasil) - Ano 2008

\begin{tabular}{|c|c|c|}
\hline \multicolumn{2}{|c|}{ Variáveis } & Descrição - Valor bruto da produção agropecuária (em reais) \\
\hline \multirow{8}{*}{$\begin{array}{l}\text { Valor } \\
\text { bruto da } \\
\text { produção } \\
\text { VBPi }\end{array}$} & $V B P_{1}$ & $\begin{array}{l}\text { Olerícola: abóbora, abobrinha, alface, batata, batata-doce, beterraba, cebola, } \\
\text { cenoura, mandioca para mesa, pimentão, repolho e tomate para mesa (Grupo de } \\
\text { produtos OLE). }\end{array}$ \\
\hline & $V B P_{2}$ & $\begin{array}{l}\text { Produtos animais: carne bovina, carne de frango, carne suína, casulo, leite B, leite C, } \\
\text { mel e ovos (Grupo PAN). }\end{array}$ \\
\hline & $V B P_{3}$ & Frutas frescas de culturas temporárias: abacaxi, melancia e morango (Grupo FRFT). \\
\hline & $\mathrm{VBP}_{4}$ & $\begin{array}{l}\text { Frutas frescas de culturas perenes: abacate, banana, caqui, figo para mesa, goiaba } \\
\text { para mesa, laranja para mesa, limão, manga, maracujá, pêssego para mesa, } \\
\text { tangerina e uva para mesa (Grupo FRFP). }\end{array}$ \\
\hline & $\mathrm{VBP}_{5}$ & $\begin{array}{l}\text { Produtos vegetais para a indústria de culturas temporárias: cana-de-açúcar, } \\
\text { mandioca e tomate (Grupo GRFT). }\end{array}$ \\
\hline & $\mathrm{VBP}_{6}$ & $\begin{array}{l}\text { Produtos vegetais para a indústria de culturas perenes: borracha, café beneficiado, } \\
\text { goiaba e laranja (Grupo GRFT). }\end{array}$ \\
\hline & $V B P_{7}$ & $\begin{array}{l}\text { Grãos e fibras de culturas temporárias: amendoim, arroz, feijão, milho, soja, sorgo, } \\
\text { trigo e triticale (Grupo GRFP). }\end{array}$ \\
\hline & $V B P_{8}$ & Grãos e fibras de culturas perenes: algodão (Grupo PVIT). \\
\hline Variáveis & & Descrição - Áreas rurais (ha) \\
\hline \multirow{3}{*}{ Área rural } & APE & Área com cultura perene compreende as terras ocupadas com lavouras perenes. \\
\hline & ATE & $\begin{array}{l}\text { Área com cultura temporária compreende as terras ocupadas com lavouras } \\
\text { temporárias (também conhecidas como anuais). }\end{array}$ \\
\hline & APA & $\begin{array}{l}\text { Área com pastagem compreende terras ocupadas com capins e similares, } \\
\text { efetivamente utilizadas na exploração animal, incluindo as destinadas a capineiras } \\
\text { e fornecimento de matéria verde para silagem ou feno. }\end{array}$ \\
\hline
\end{tabular}

Fonte: Instituto de Economia Agrícola (2018) e São Paulo (2009) com elaboração própria. 
A descrição de cada variável pode ser observada na tabela 1. As oito primeiras variáveis são relacionadas ao valor bruto de produção (VBP), em reais (R\$), por conjuntos de produtos (, com $i=1, \ldots 8$ tipos de produtos), adaptadas a partir da metodologia empregada pelo Instituto de Economia Agrícola do Estado de São Paulo (Instituto de Economia Agrícola, 2018), com separação adicional em culturas temporárias e perenes. Na sequência, observam-se três variáveis relacionadas à forma de utilização das áreas rurais, em hectares (ha), segundo o projeto de Levantamento Censitário das Unidades de Produção Agropecuária do Estado de São Paulo (LUPA) de 2007/2008 (São Paulo, 2009).

Nesta pesquisa, foi utilizada a produtividade da terra, em unidades monetárias, como critério para se hierarquizar os municípios com atividade agropecuária no estado de São Paulo, sendo que as variáveis relacionadas ao valor bruto de produção foram atualizadas pelo índice de preços ICP-M entre dezembro de 2008 e dezembro de 2019.

As áreas rurais com cultura perene $(A P E)$, temporária $(A T E)$ e pastagem $(A P A)$ foram somadas, resultando na área produtiva total, ou seja, $P T=A P E+A T E+A P A$.

Dessa forma, a partir do vetor aleatório $V B P$, formado por oito variáveis $V B P_{I}$ (com $i=1, \ldots 8$ conjuntos de produtos), representando respectivamente o valor bruto de produção de cada conjunto de produtos (OLE, PAN, FRFT, FRFP, GRFT, GRFP, PVIT e PVIP), constituiu-se um novo vetor aleatório, denominado de contribuição financeira por hectare $(C F)$, formado por novas variáveis $C F_{i}$ relacionando os $V B P_{i}$ e a área produtiva total do município (APT).

Assim, para o j-ésimo município, a contribuição financeira por hectare do $i$-ésimo conjunto de produtos é obtida por:

$$
C F_{i j}=\frac{V B P_{i j}}{A P T_{j}}
$$

sendo $C F_{i j}$ a contribuição financeira por hectare do conjunto de produtos $i$ no município $j$ (em R $\$ . h a^{-1}$ ), $V B P_{i j}$ o valor bruto da produção do conjunto de produtos $i$ $\left(\mathrm{R} \$\right.$ ) no município $j$ e $A P T_{j}$ a área produtiva total útil do município $j$ (ha).

A contribuição financeira por hectare total de cada município $\left(C F T_{j}\right)$ é o somatório das $C F_{i j}$ de cada conjunto de produtos de um município, ou seja:

$$
C F T_{j}=\sum_{i=1}^{8} C F_{i j}=\frac{\sum_{i=1}^{8} V B P_{i j}}{A P T_{j}}
$$

correspondendo à produtividade total por hectare do município.

Assim, um indicador da participação da contribuição financeira ou índice de concentração $\left(P C F_{i j}\right)$ de cada conjunto de produtos na contribuição financeira total do município $j$, foi obtido pela razão entre a $C F_{i j}$ e $C F T_{j}$, para $i=1, \ldots 8$ indicando 
respectivamente os grupos de produtos $\operatorname{OLE}\left(P C F_{1 j}\right)$, PAN $\left(P C F_{2 j}\right)$, FRFT $\left(P C F_{3 j}\right)$, FRFP $\left(P C F_{4 j}\right)$, PVIT $\left(P C F_{5 j}\right)$, PVIP $\left(P C F_{6 j}\right)$, GRFT $\left(P C F_{7 j}\right)$ e GRFP $\left(P C F_{8 j}\right)$.

Desta forma, para um indicativo de concentração de um determinado conjunto de produtos em um município foi considerado quando a participação $\left(P C F_{i j}\right)$, representasse no mínimo 0,5 , em uma escala de 0 a 1 , indicando que o conjunto de produtos em questão concentra pelo menos metade da contribuição financeira por hectare total $\left(C F T_{j}\right)$ do município. Mais duas medidas de concentração foram calculadas para cada município: a razão de concentração $\left(C R_{j}\right)$ e o índice de Hirschman-Herfindahl $\left(H H I_{j}\right)$ adaptados para os conjuntos de produtos seguindo a descrição apresentada por Resende (1994).

A razão de concentração foi adaptada para os conjuntos de produtos de maior participação no estado de São Paulo, ou seja, PAN e PVIT. Seu resultado deve variar entre o (zero) e 1 (um), onde "o" representa uma situação de inexistência destes conjuntos no município $j$; e "1" indica uma condição de concentração intensa. Esse indicador da razão de concentração é representado da seguinte forma:

$$
C R_{j}=\sum_{i=1}^{k} C F_{i j}=P_{i j} \text { em que, }
$$

$k$ representa a quantidade de conjuntos (PAN e PVIT para este estudo), sendo, assim, considerado $k=2$ e $P_{i j}$ e corresponde a participação de cada conjunto de produtos no município ( $i$ igual a 1 ou 2 e $j$ de 1 até o número de municípios avaliados). Dada a variação do resultado do índice $C R_{i j}$ entre "O" e "1", é necessário ponderar o resultado dentro dessa escala. No texto do trabalho $C R_{i}$, foi tratado por $C R$.

O segundo indicador, adaptado do Índice de Hirchsman-Herfindahl $\left(H H I_{j}\right)$, obtido pela soma dos quadrados da participação dos conjuntos de produtos considerados. Tem-se, assim:

$$
H H I_{j}=\sum_{i=1}^{k}\left(P_{i j}\right)^{2} \text { em que, }
$$

$k$ representa os conjuntos de produtos, sendo considerado neste indicador os oito conjuntos avaliados inicialmente (ou seja, $k=8$ ) e $P_{i j}$ representa a participação de cada conjunto de produtos $(i=1, \ldots 8)$ no município $j$. A expressão reverencia de forma implícita os pesos desse indicador ao submeter cada parcela do conjunto de produtos ao quadrado, ela atribui maior grau de concentração aos maiores conjuntos. Existem escalas para determinar o nível de concentração deste índice, contudo, por se tratar de apenas oito conjuntos, este indicador de concentração considerou a comparação entre os resultados dos conjuntos, tendo em vista que de outra forma haveria sempre o resultado de "alta concentração".

Os indicadores dos grupos de municípios formados a partir dos conjuntos de produtos que foram apresentados em tabelas, sem o índice para indicar o municí- 
pio, sendo representados respectivamente por $P C F_{i}(i=1, \ldots 8), C F T, C R$ e $H H I$, e os grupos de município resumidos por meio das estatísticas descritivas: média, desvio-padrão, coeficiente de variação e percentis, 80, 50 e 20.

\section{Resultados e discussão}

A contribuição financeira por hectare (CFT) do estado de São Paulo foi de $\mathrm{R} \$ 2.407,41$, com sua distribuição por conjuntos de produtos representados na figura 1. Pode-se perceber que o conjunto de maior expressão foi o de produtos vegetais para indústria de culturas temporárias (PVIT), com R \$892,19 (37\%) por hectare, seguido pelos produtos de origem animal (PAN), com $\mathrm{R} \$ 648,27$ (27\%).

Figura 1. Contribuição financeira por hectare (CFT) dos conjuntos de produtos no estado de São Paulo em 2008 (em reais e percentual de participação na contribuição total)

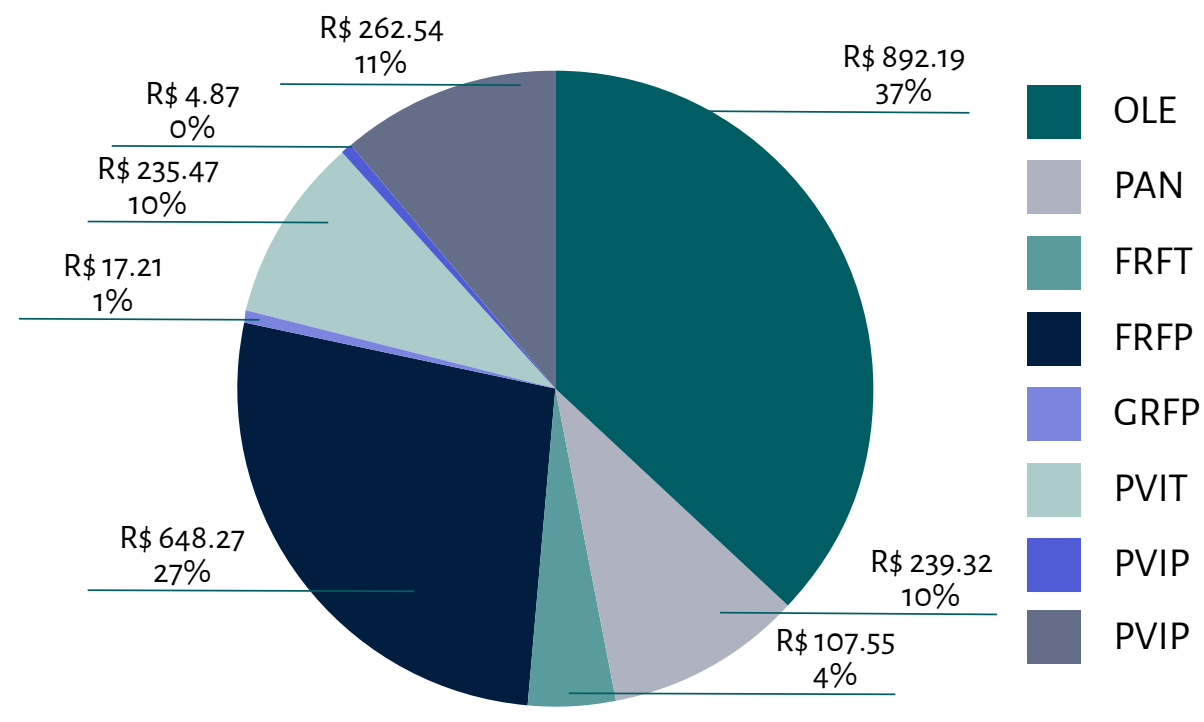

Fonte: Instituto de Economia Agrícola. (2018) e São Paulo (2009) com elaboração própria.

O conjunto dos produtos olerícolas (OLE) foi composto apenas por culturas temporárias, com um índice de concentração médio de 0,70 nos 14 municípios em que $P C F_{1}$ foi o conjunto de maior concentração produtiva, como se verifica na tabela 2. Nestes munícipios a razão de concentração em PVIT e PAN foi de 0,15 (CR) e houve alta concentração produtiva de 0,56 (HHI), tendo em vista que restaram apenas 0,15 da produção destes municípios nos demais conjuntos. A média produtiva destes municípios foi superior ao estado $(\mathrm{R} \$ 4.704,31)$. A mediana indica que pelo menos a metade dos municípios tiveram CFT acima da média $(R \$ 5189,31)$. O destaque do 
conjunto foram as hortaliças, pois as tendências dos mercados globalizados apontam perspectivas favoráveis para as hortaliças exigindo que a uniformidade desses produtos seja cada vez mais obrigatória (Vilela e Macedo, 2000). No que diz respeito às olerícolas de um modo geral, trata-se de culturas de ciclo curto e exigentes quanto ao uso de insumos, irrigação, adubação e tratos culturais, apresentam uma estacionalidade bem definida, que provoca queda nos preços e consequentemente depressão na renda do produtor (Rodrigues, Martins e Araújo, 1997).

Tabela 2. Resumo numérico da participação da contribuição financeira ou índice de concentração $\left(\mathrm{PCF}_{1}\right)$ do conjunto de produtos olerícolas (OLE), contribuição financeira total (CFT), razão de concentração (CR) e índice de Hirchsman-Herfindahl (HHI) em 2008

\begin{tabular}{|l|l|l|l|l|}
\multicolumn{1}{|c|}{ Indicador } & \multicolumn{1}{c|}{ PCF $_{1}$} & \multicolumn{1}{c|}{ CFT (R\$) } & \multicolumn{1}{c|}{ CR } & \multicolumn{1}{c|}{ HHI } \\
\hline Média & 0,70 & $4.704,31$ & 0,15 & 0,56 \\
\hline Desvio & 0,15 & $2.938,19$ & 0,11 & 0,18 \\
\hline CV (\%) & 21,49 & 62,46 & 72,11 & 32,83 \\
\hline Max & 0,99 & $9.916,03$ & 0,37 & 0,98 \\
\hline Min & 0,51 & 932,92 & 0,00 & 0,36 \\
\hline P80 & 0,82 & $6.552,24$ & 0,25 & 0,69 \\
\hline P50 & 0,67 & $5.189,31$ & 0,14 & 0,52 \\
\hline P20 & 0,54 & $1.137,68$ & 0,06 & 0,39 \\
\hline 14 (2,3\%) municípios & & & & \\
\hline
\end{tabular}

Fonte: Instituto de Economia Agrícola. (2018) e São Paulo (2009) com elaboração própria.

Os produtos de origem animal (PAN) apresentaram maior participação na contribuição financeira $\left(P C F_{2}\right)$ em 169 municípios (tabela 3). A média da CFT foi de $\mathrm{R} \$ 2.523,00$, porém pelo menos $50 \%$ dos municípios tiveram a CFT de até $R \$ 1.188,87$ e com o valor do percentil 80 foi pouco superior à média $(R \$ 2.934,11)$. Portanto, os $20 \%$ de municípios com maior CFT apresentaram alto desempenho produtivo índice de concentração de 0,91. O CR médio de 0,83 indica pouca participação de PVIT $(0,11)$ e demais produtos justificando al ta concentração com HHI de o,60.

$\mathrm{O}$ produto de origem animal de maior participação foi a carne bovina com 50,8 \% (Silva et al., 2020). A principal característica no desenvolvimento dessa atividade no país é a heterogeneidade nos sistemas de produção e nos mecanismos de gestão e de comercialização do gado. Coexistem dois subsistemas de produção bastante distintos. O primeiro subsistema de alta qualidade, caracterizado pela adoção de tecnologia avançada e padrões eficientes de gestão e de comercialização. O segundo, de baixa qualidade, baseia-se na produção extensiva, pequena intensidade tecnológica e padrões precários de gestão e de comercialização do gado bovino (Carvalho e Zen, 2017). A dicotomia entre sistemas de alta e baixo padrão pode explicar a grande variabilidade do CFT dos produtos de origem animal. 
Tabela 3. Resumo numérico da participação da contribuição financeira ou índice de concentração $\left(\mathrm{PCF}_{2}\right)$ do conjunto de produtos de origem animal (PAN), contribuição financeira total (CFT), razão de concentração (CR) e índice de Hirchsman-Herfindahl (HHI) em 2008

\begin{tabular}{|c|c|c|c|c|}
\hline Indicador & $P C F_{2}$ & CFT $(R \$)$ & CR & $\mathrm{HHI}$ \\
\hline Media & 0,72 & $2.523,00$ & 0,83 & 0,60 \\
\hline Desvio & 0,16 & $4.517,22$ & 0,13 & 0,21 \\
\hline CV (\%) & 21,80 & 179,04 & 15,40 & 34,37 \\
\hline Max & 1,00 & $32.141,92$ & 1,00 & 1,00 \\
\hline Min & 0,50 & 63,21 & 0,50 & 0,32 \\
\hline P80 & 0,91 & $2.934,11$ & 0,95 & 0,83 \\
\hline P50 & 0,70 & $1.188,87$ & 0,84 & 0,53 \\
\hline P2O & 0,57 & 708,60 & 0,72 & 0,42 \\
\hline \multicolumn{5}{|c|}{ 169(27,2\%) municípios } \\
\hline
\end{tabular}

Fonte: Instituto de Economia Agrícola. (2018) e São Paulo (2009) com elaboração própria.

$\mathrm{Na}$ tabela 4 se observa que os municípios cujo principal conjunto foi frutas frescas de culturas perenes (maior PCF4), apresentaram a mais alta concentração produtiva $(0,82)$ no estado. O valor médio da CFT $(\mathrm{R} \$ 8.739,14)$ foi superior ao percentil 80 indicando alto desempenho de alguns municípios em relação à maioria restante, que se expressa em uma heterogeneidade mostrada no CV de 146,13\%. Este conjunto destaca-se como o de maior desempenho por hectare no estado em todas as faixas de análise.

O PVIT e o PAN tiveram pouca participação média nestes municípios (CR igual a 0,12 ), mas com alta concentração em poucos conjuntos no estado ( $\mathrm{HHI}$ de 0,73$)$. $\mathrm{O}$ conjunto de frutas frescas apresentou o maior CFT médio entre os conjuntos e assim como constataram Petinari, Tereso e Bergamasco (2008), a fruticultura mostrou-se como alternativa para pequenas áreas, e as propriedades onde predomina essa atividade, desde que bem conduzidas, são capazes de manter toda a família trabalhando e conseguindo obter um bom retorno financeiro. Neste sentido, as desigualdades regionais quanto à área produtiva e a gestão das propriedades, bem como o uso de tecnologias visando ganho de produtividade e oferta de trabalho, são significativas mesmo no âmbito de pequenos produtores familiares (Belik, 2015), no entanto, deve-se destacar que na agricultura em pequenas áreas observam-se desde produtores familiares com bons resultados financeiros até a situação de produção para subsistência (Souza, Ney, Fornazier e Ponciano, 2015). 
Tabela 4. Resumo numérico da participação da contribuição financeira ou índice de concentração $\left(\mathrm{PCF}_{4}\right)$ do conjunto de frutas frescas e culturas permanentes (FRFP), contribuição financeira total (CFT), razão de concentração (CR) e índice de Hirchsman-Herfindahl (HHI) em 2008

\begin{tabular}{|l|r|r|r|r|}
\hline Indicador & \multicolumn{1}{|c|}{$\mathrm{PCF}_{4}$} & $\mathrm{CFT}(\mathrm{R} \$)$ & \multicolumn{1}{c|}{$\mathrm{CR}$} & $\mathrm{HHI}$ \\
\hline Média & 0,82 & $8.739,14$ & 0,12 & 0,73 \\
\hline Desvio & 0,16 & $12.770,77$ & 0,11 & 0,21 \\
\hline CV (\%) & 19,37 & 146,13 & 95,08 & 28,52 \\
\hline Max & 1,00 & $55.658,13$ & 0,39 & 1,00 \\
\hline Min & 0,53 & 269,56 & 0,00 & 0,37 \\
\hline P80 & 0,96 & $8.644,85$ & 0,25 & 0,92 \\
\hline P50 & 0,89 & $4.690,95$ & 0,08 & 0,80 \\
\hline P20 & 0,65 & $1.973,82$ & 0,03 & 0,49 \\
\hline 36(5,8\%) municípios & & & \\
\hline
\end{tabular}

Fonte: Instituto de Economia Agrícola. (2018) e São Paulo (2009) com elaboração própria.

O conjunto de municípios com maior índice de concentração de PVIT $\left(P C F_{5}\right)$, apresentado na tabela 5, apresentou a maior quantidade de municípios (191), com índice de concentração médio de 0,69 , com 0,17 de PAN e 0,14 nos demais conjuntos. A média e a mediana ( $\left(P_{50}\right)$ estavam próximas ( $\mathrm{R} \$ 2.507,78$ e $\mathrm{R} \$ 2.532,72$ ) com o menor coeficiente variação entre os conjuntos analisados (29,37\%). Entre os produtos que compõem o PVIT a cana de açúcar representou 36,66 \% do valor da produção paulista (Silva et al., 2020) e 95,1 \% do conjunto.

Portanto percebe-se que o comportamento do conjunto representado pela cana de açúcar, foi o de maior importância econômica pela sua abrangência e também o mais homogêneo por possuir a menor variabilidade.

No percentil 80 houve a maior concentração do PVIT e do CR, enquanto nos percentis 50 e 20 reduziu a concentração em PVIT $(0,67$ e 0,57) com aumento na participação de PAN, pois o CR decresceu em menor proporção. Desta forma, percebe-se uma presença complementar do PAN, nos municípios de concentração de PVIT.

Os principais produtos do conjunto de municípios (1,8\% do total de municípios do Estado) com maiores participação de PVIP na contribuição financeira $\left(P C F_{6}\right)$ foi o café beneficiado e a laranja para indústria, com 93,2 \% do conjunto. Na tabela 6, o indicador médio de CFT para os municípios de PVIP foi $4 \%$ superior ao PVIT, sendo que os demais indicadores se situaram próximos, com importante participação do PVIT e do PAN (CR igual a 0,31) complementares neste conjunto, restando apenas 0,08 para outros conjuntos de produtos. Como ocorreu no conjunto do PVIT, os valores do CFT apresentaram coeficiente de variação estável quando comparado aos demais conjuntos de produtos, indicando produtividades semelhantes nos municípios. 
Tabela 5. Resumo numérico da participação da contribuição financeira ou índice de concentração $\left(P \mathrm{CF}_{5}\right)$ do conjunto de produtos vegetais para indústria de culturas temporárias (PVIT), contribuição financeira total (CFT), razão de concentração (CR) e índice de Hirchsman-Herfindahl (HHI) em 2008

\begin{tabular}{|l|l|l|l|l|}
\hline Indicador & \multicolumn{1}{|c|}{ PCF5 } & \multicolumn{1}{|c|}{ CFT (R\$) } & \multicolumn{1}{c|}{ CR } & \multicolumn{1}{c|}{ HHI } \\
\hline Média & 0,69 & $2.507,78$ & 0,86 & 0,55 \\
\hline Desvio & 0,13 & 736,45 & 0,10 & 0,15 \\
\hline CV (\%) & 18,70 & 29,37 & 11,33 & 27,13 \\
\hline Max & 1,00 & $4.894,50$ & 1,00 & 1,00 \\
\hline Min & 0,50 & $1.013,27$ & 0,56 & 0,34 \\
\hline P80 & 0,82 & $3.115,28$ & 0,94 & 0,69 \\
\hline P50 & 0,67 & $2.532,72$ & 0,87 & 0,52 \\
\hline P20 & 0,57 & $1.825,15$ & 0,79 & 0,42 \\
\hline 191(30,75\%) municípios & & & \\
\hline
\end{tabular}

Fonte: Instituto de Economia Agrícola. (2018) e São Paulo (2009) com elaboração própria.

Tabela 6. Resumo numérico da participação da contribuição financeira ou índice de concentração $\left(\mathrm{PCF}_{6}\right)$ do conjunto de produtos vegetais para indústria de culturas perenes (PVIP), contribuição financeira total (CFT), razão de concentração (CR) e índice de Hirchsman-Herfindahl (HHI) em 2008

\begin{tabular}{|c|c|c|c|c|}
\hline Indicador & & CFT (R\$) & CR & $\mathrm{HHI}$ \\
\hline Média & 0,61 & $2.605,78$ & 0,31 & 0,46 \\
\hline Desvio & 0,10 & 963,10 & 0,11 & 0,09 \\
\hline CV (\%) & 15,97 & 36,96 & 34,14 & 19,27 \\
\hline Max & 0,80 & $4.938,02$ & 0,46 & 0,65 \\
\hline Min & 0,50 & $1.655,11$ & 0,13 & 0,37 \\
\hline P80 & 0,68 & $3.047,24$ & 0,40 & 0,52 \\
\hline P50 & 0,59 & $2.288,43$ & 0,34 & 0,43 \\
\hline P2O & 0,52 & $2.131,58$ & 0,22 & 0,39 \\
\hline \multicolumn{5}{|c|}{ 11(1,8\%) municípios } \\
\hline
\end{tabular}

Fonte: Instituto de Economia Agrícola. (2018) e São Paulo (2009) com elaboração própria.

Os indicadores dos municípios com maiores participações do conjunto de grãos e fibras tiveram um CFT médio de $\mathrm{R} \$ 3.290,65$ com, pelo menos, $50 \%$ dos municípios com CFT de $R \$ 3.411,20$ e índice de concentração médio de 0,62 (tabela 7). O desempenho por hectare esteve abaixo apenas do FRFP $(R \$ 8.739,14)$ e OLE ( $R \$ 4.704,31$ ), com mediana do CFT acima da média demonstrou que mais de $50 \%$ dos municípios estavam acima da média. Os principais produtos do conjunto fo- 
ram o milho $(46,6 \%)$, a soja $(21,6 \%)$ e feijão $(20,1 \%)$ representando $88,3 \%$. O uso destes produtos, para alimentação animal e humana, contribui para o valor da CFT. Segundo Zambom et al. (2008) a utilização do milho e da soja na alimentação de aves e suínos tem-se intensificado no mundo o que eleva seus preços, assim como derivados destas fibras na alimentação de bovinos. Na alimentação humana, Maluf e Speranza (2014) destacam o consumo massivo do feijão no Brasil, sendo responsável em parte pela inflação de alimentos. Além disto, o Brasil passou de exportador para importador de feijão mesmo com a redução do seu consumo nos últimos anos.

Tabela 7. Resumo numérico da participação da contribuição financeira ou índice de concentração $\left(\mathrm{PCF}_{7}\right)$ do conjunto de grãos e fibras de culturas temporárias (GRFT), contribuição financeira total (CFT), razão de concentração (CR) e índice de Hirchsman-Herfindahl (HHI) em 2008

\begin{tabular}{|l|r|r|r|r|}
\hline \multicolumn{1}{|c|}{ Indicador } & \multicolumn{1}{c|}{ PCF7 } & \multicolumn{1}{c|}{ CFT $(\mathbf{R} \$)$} & \multicolumn{1}{c|}{ CR } & \multicolumn{1}{c|}{ HHI } \\
\hline Média & 0,62 & $3.290,65$ & 0,28 & 0,49 \\
\hline Desvio & 0,13 & $1.340,82$ & 0,13 & 0,13 \\
\hline CV (\%) & 20,43 & 40,75 & 46,19 & 27,36 \\
\hline Max & 0,87 & $5.659,76$ & 0,49 & 0,77 \\
\hline Min & 0,51 & 622,86 & 0,11 & 0,33 \\
\hline P80 & 0,71 & $4.088,86$ & 0,43 & 0,58 \\
\hline P50 & 0,56 & $3.411,20$ & 0,26 & 0,46 \\
\hline P20 & 0,52 & $2.554,60$ & 0,15 & 0,36 \\
\hline $16(2,6 \%)$ municípios & & \\
\hline
\end{tabular}

Fonte: Instituto de Economia Agrícola. (2018) e São Paulo (2009) com elaboração própria.

O grupo de 86 municípios da tabela 8 precisou dos conjuntos $\mathrm{PAN}\left(\mathrm{PCF}_{2}\right.$ médio de 0,32$)$ e PVIT $\left(P C F_{5}\right.$ médio de 0,34$)$ para atingir uma razão de concentração média acumulada $\left(P C F_{\text {total }}=P C F_{2}+P C F_{5}\right)$ acima de $50 \%$. OPCF total de 0,66 representa o somatório da concentração de PAN mais PVIT. Portanto, considerando-se apenas os dois conjuntos de produtos, 446 municípios (71,82 \%) tiveram PAN e PVIT como principais conjuntos.

O CFT dos municípios que tem PAN como produto principal se assemelha ao conjunto PVIT ( $R \$ 2.523,00$ e $R \$ 2.507,78$ respectivamente) sendo um pouco abaixo quando agrupados ( $R \$ 2.227,07)$, sugerindo menor tecnificação em municípios com menor concentração. $O$ valor mediano de $P C F_{2}$ (conjunto PAN) foi $52,87 \%$ abaixo da média, enquanto no $\mathrm{PCF}_{5}$ (conjunto PVIT) foi $1 \%$ acima da média e os agrupamentos dos dois conjuntos foi $14,2 \%$ abaixo.

Desta forma, os municípios com PVIT possui uma distribuição mais homogênea da produção, enquanto a produtividade dos municípios de PAN demonstrou-se menos homogênea no estado. Houve aumento da diversificação produtiva $(\mathrm{HHI}$ de o,32) com PAN e PVIT como principais produtos e 0,34 em outros conjuntos. Esta 
distribuição produtiva não contribuiu para aumentar a CFT, sendo esta a menor nos grupos de municípios do estado.

A decisão de especializar ou diversificar a produção é influenciada por vários fatores. Além das vantagens e das desvantagens econômicas de adotar cada tipo de produção, diferentes oportunidades e limitações podem afetar a decisão dos produtores (Schroth e Ruf, 2014).

Tabela 8. Resumo numérico da participação da contribuição financeira ou índice de concentração $\left(\mathrm{PCF}_{2}\right.$ e $\mathrm{PCF}_{5}$ ) dos conjuntos de produtos de origem animal (PAN) e produtos vegetais para indústria de culturas temporárias (PVIT), contribuição financeira total (CFT), razão de concentração (CR) e índice de Hirchsman-Herfindahl (HHI) em 2008

\begin{tabular}{|l|r|r|r|r|r|}
\hline \multicolumn{1}{|c|}{ Indicador } & \multicolumn{1}{c|}{$\mathrm{PCF}_{2}$} & \multicolumn{1}{c|}{$\mathrm{PCF}_{5}$} & \multicolumn{1}{c|}{$\mathrm{CFT}(\mathrm{R} \$)$} & \multicolumn{1}{c|}{$\mathrm{CR}$} & \multicolumn{1}{c|}{$\mathrm{HHI}$} \\
\hline Média & 0,32 & 0,34 & $2.227,07$ & 0,66 & 0,32 \\
\hline Desvio & 0,13 & 0,12 & $1.071,20$ & 0,12 & 0,05 \\
\hline CV (\%) & 40,42 & 35,13 & 48,10 & 17,80 & 15,49 \\
\hline Max & 0,49 & 0,49 & $6.233,96$ & 0,96 & 0,46 \\
\hline Min & 0,04 & 0,04 & 180,99 & 0,50 & 0,22 \\
\hline P80 & 0,45 & 0,45 & $3.077,42$ & 0,77 & 0,36 \\
\hline P50 & 0,36 & 0,38 & $1.910,66$ & 0,64 & 0,32 \\
\hline P20 & 0,18 & 0,23 & $1.410,04$ & 0,55 & 0,28 \\
\hline 86 (13,85\%) municípios & & & & \\
\hline
\end{tabular}

Fonte: Instituto de Economia Agrícola. (2018) e São Paulo (2009) com elaboração própria.

Para atingir no mínimo 50 \% no acumulado dos índices de concentração nos municípios da tabela 9, foi necessário agrupar três conjuntos de produtos: PAN, FRFP e PVIP (com índice de concentração acumulado $P C F_{\text {total }}=P C F_{2}+P C F_{4}+P C F_{6}$ igual a 0,66 ). O CR foi de 0,33 , portanto o $+P C F_{5}$ de PVIT (incluído no $C R$ ) foi de 0,08 , pois o PAN está incluído no CR. Esta distribuição produtiva fez com que a concentração global em conjuntos de produtos fosse a menor de todas (HHI de 0,33). Ao avaliar os índices de concentração acumulado de quatro conjuntos de produtos (PAN, FRFP, PVIP e o PVIT) com diferentes características de CFT elevou-se o coeficiente de variação que atingiu 115,59 \%. A média do CFT de $\mathrm{R} \$ 3.471,34$ foi superior a PAN e PVIP ( $R \$ 2.523,00$ e $R \$ 2.605,78$ respectivamente), contudo inferior a FRFP $(R \$ 8.739,14)$. A mediana inferior à média indica que houve municípios com desempenho muito elevados nos $50 \%$ mais produtivos. Para a diversificação como forma de melhorar o desempenho produtivo é fundamental a disponibilidade de assistência técnica qualificada para a promoção da diversificação produtiva (Singha, Baruah, Bordoloi, Dutta e Saikia, 2012). 
Tabela 9. Resumo numérico do índice de concentração acumulado $\left(\mathrm{PCF}_{2}, \mathrm{PCF}_{4} \mathrm{e} P \mathrm{PF}_{6}\right)$ dos conjuntos de produtos PAN, FRFP e FVIP, contribuição financeira total (CFT), razão de concentração (CR) e índice de Hirchsman-Herfindahl (HHI) em 2008

\begin{tabular}{|l|r|r|r|r|r|r|r|}
\hline Indicador & $\mathrm{PCF}_{2}$ & $\mathrm{PCF}_{4}$ & $\mathrm{PCF}_{6}$ & $\mathrm{PCF}_{\text {total }}$ & \multicolumn{1}{c|}{ CFT(R) } & \multicolumn{1}{c|}{$\mathrm{CR}$} & HHI \\
\hline Média & 0,25 & 0,20 & 0,21 & 0,66 & $3.471,34$ & 0,33 & 0,38 \\
\hline Desvio & 0,15 & 0,14 & 0,15 & 0,21 & $4.012,59$ & 0,15 & 0,19 \\
\hline CV (\%) & 59,20 & 68,75 & 71,50 & 31,60 & 115,59 & 46,13 & 50,96 \\
\hline Max & 0,50 & 0,50 & 0,50 & 0,99 & $37.846,39$ & 0,95 & 1,00 \\
\hline Min & 0,00 & 0,00 & 0,00 & 0,15 & 841,27 & 0,00 & 0,13 \\
\hline P80 & 0,40 & 0,34 & 0,35 & 0,86 & $4.038,31$ & 0,45 & 0,46 \\
\hline P50 & 0,24 & 0,20 & 0,22 & 0,64 & $2.831,65$ & 0,35 & 0,32 \\
\hline P20 & 0,10 & 0,06 & 0,05 & 0,51 & $1.767,61$ & 0,18 & 0,26 \\
\hline 98 (15,78\%) municípios & & & & & \\
\hline
\end{tabular}

Fonte: Instituto de Economia Agrícola. (2018) e São Paulo (2009) com elaboração própria.

No caso da agricultura familiar, destaca-se também a importância de se ter a assistência técnica pública gratuita, para que os agricultores descapitalizados não fiquem reféns da assistência dada por empresas produtoras de insumos agropecuários, as quais visam vender os seus produtos e não têm interesse em promover sistemas diversificados que tendem a usar menos insumos químicos em sua produção (Sambuichi, Galindo, Oliveira e Pereira, 2014).

O conjunto de produtos FRFT (abacaxi, melancia e morango) foi o principal produto no município de Guaraçai $\left(\mathrm{PCF}_{3}\right.$ igual a 0,44$)$ e GFRP (algodão) em Paranapanema $\left(P C F_{8}\right.$ de 0,42$)$ ambos abaixo de 0,50 , portanto sem concentração suficiente para a formação de grupos de municípios de conjunto único ou associados a outros conjuntos.

\section{Considerações finais}

Os produtos vegetais para indústria foi a principal atividade agropecuária em 202 municípios do estado de São Paulo, sendo 191 em culturas temporárias predominadas pela cana de açúcar e em 11 por culturas perenes, principalmente pelo café e pela laranja para indústria. Estes produtos são commodities agrícolas com demanda por complexos agroindustriais favorecendo a opção pela sua produção. As contribuições financeiras por hectare destes conjuntos estiveram bastante próximas, com a menor variabilidade financeira nos municípios entre todos os conjuntos de produtos analisados. A proximidade de rentabilidade por hectare, entre os principais produtos vegetais, faz com que o custo de oportunidade de mudança de uma cultura para outra se minimize no longo prazo. 
Os municípios que tiveram os produtos de origem animal como principal conjunto apresentaram contribuição financeira média semelhante aos produtos vegetais para indústria, contudo com alta variabilidade, com grandes diferenças de rentabilidade e características produtivas bastante heterogêneas. As diferenças de rentabilidade observadas foram bastante significativas com os menores valores por hectare útil, sugerindo a necessidade de políticas públicas direcionadas a esses produtores a fim de aumentar a eficiência e consequentemente a rentabilidade.

Os conjuntos de produtos olerícolas e de frutas frescas apresentaram as maiores médias de contribuição financeira por hectare e representaram aproximadamente $15 \%$ do valor bruto da produção paulista. A perecibilidade, a intensividade de mão de obra e a demanda de mercado são entraves para expansão destes conjuntos de produtos, mesmo com o atrativo da rentabilidade por hectare.

O conjunto de grãos e fibras em culturas temporárias, com destaque para a soja, milho e feijão, apresentaram contribuição financeira e variabilidade intermediária entre os conjuntos, superior aos produtos vegetais para indústria. Existe demanda por estes produtos no mercado interno e externo, contudo há necessidade de áreas extensas para que os custos de produção se reduzam e consequentemente aumente a lucratividade. A produção destes municípios foi complementada principalmente por produtos vegetais para indústria e produtos de origem animal.

O grau de especialização produtiva por conjunto de produtos demonstrou uma maior concentração produtiva acompanhada por maior rentabilidade por hectare. O forte dilema entre diversificação para diluir os riscos de mercado e a especialização para aumentar a eficiência produtiva foi verificado em todos os conjuntos de produtos. Nos municípios em que houve vários conjuntos de produtos para atingir $50 \%$ da rentabilidade por hectare, ocorreu redução de rentabilidade, comparado a municípios que concentravam estes mesmos produtos em mais de $50 \%$.

A opção pela diversificação incorre na necessidade de aumento de recursos financeiros e materiais para a produção com rentabilidade e consequentemente retorno para os produtores, além disso, externalidades positivas relacionadas à disponibilidade de mão de obra, fornecedores especializados e demanda de mercado contribuem para a concentração produtiva.

Por fim, vale destacar que a falta de dados mais atualizados impossibilitou avaliações de tendências e, consequentemente, se os dados podem expressar situações de anos típicos ou excepcionais de algumas produções, bem como, considerando as transformações que envolveram a atividade agrícola nos países do cone sul nos últimos anos pode ter alterado à dinâmica atual da atividade agrícola nessa região. No entanto, os indicadores propostos trazem uma contribuição para a avaliação e melhor gestão de políticas públicas voltadas ao desenvolvimento em nível local, podendo ser aplicado em qualquer região com disponibilização de dados de produção. 


\section{Referências bibliográficas}

ABRAMOVAY, R. (2003). O futuro das regiões rurais. UFRGS Editora.

ADELMAN, I. (2002). Falacias en la teoría del desarrollo y sus implicaciones en política.

Fronteras de la Economía en Desarrollo. Bogotá, Colombia: Banco Mundial-Alfa Omega, 91-124. Recuperado de: http://www3.uah.es/econ/Economia_del_ Desarrollo/Introduccion/Adelman.pdf

http://documents1.worldbank.org/curated/pt/765591468762299144/

pdf/217470Spanish-verofronteras.pdf

ARAÚJO, A.S.D. (2013). Concentração espacial e especialização do mercado de trabalho formal do Rio Grande do Norte no período (2000-2010). Dissertação (Mestrado em Economia) - Universidade Federal da Paraíba, Paraíba. Recuperado de: http:// bdtd.biblioteca.ufpb.br/handle/tede/4999. Acesso em: O2 fev. 2017.

BELIK, W. (2015). A heterogeneidade e suas implicações para as políticas públicas no rural brasileiro. Revista de Economia e Sociologia Rural, 53(1), 9-30. https://doi. org/10.1590/1234-56781806-9479005301001

CARVALHO, T.B. \& ZEN, S. (2017). A cadeia de Pecuária de Corte no Brasil: evolução e tendências. RevistaiPecege,3(1), 85-99. https://doi.org/10.22167/r.ipecege.2017.1.85 COMBES, P.P.; DURANTON, G. \& COBILLON, L. (2008). Spatial wage disparities: Sorting matters! Journal of urban economics, 63(2), 723-742. https://doi.org/10.1016/j. jue.2007.04.004

FERREIRA, P.C. \& ELLERY Jr., R. (1996). Crescimento econômico, retornos crescentes e concorrência monopolista. Brazilian Journal of Political Economy, 16(2). Retrieved from https://rep.org.br/rep/index.php/journal/article/view/1204

GASQUES, J.C.; BASTOS, E.T.; VALDES, C. \& BACCHI, M. (2013). Produtividade e crescimento: algumas comparações. Em Alves, E.R.A.; Souza, G.S. \& Comes, E.C, Contribuição da Embrapa para o desenvolvimento da agricultura no Brasil (155-140). Brasília, Brasil: Embrapa.

HADDAD, P.R. (1989). Medidas de localização e de especialização. Economia regional: teorias e métodos de análise. Fortaleza: BNB-ETENE, 225-248.

INSTITUTO DE ECONOMIA AGRÍCOLA (2018). Banco de dados. Solicitação especial estatísticas de produção agropecuária municipal e valor bruto da produção. 2018.

LIMA, A.C.D.C. \& SIMÕES, R.F. (2010). Teorias clássicas do desenvolvimento regional e suas implicações de política econômica: o caso do Brasil. RDE-Revista de Desenvolvimento Econômico, 12(21).

LIU, G., MIRZAEI, A. \& VANDOROS, S. (2014). The impact of bank competition and concentration on industrial growth. Economics Letters, 124(1), 60-63. https://doi. org/10.1016/j.econlet.2014.04.016

MALUF, R.S. \& SPERANZA, J.S. (2014). Preços dos alimentos, modelos de agricultura e abastecimento alimentar no Brasil: os casos da soja e do feijão. Relatório Técnico, 
7. Recuperado de: http://oppa.net.br/acervo/documentos/CERESAN \%20 Relat \%20Tecnico \%207\%20Estudo \%20precos \%20AAid \%20(2).pdf MEDEIROS COSTA, C.C.; FERREIRA, M.A.M.; BRAGA, M.]. \& ABRANTES, L. A. (2012). Disparidades inter-regionais e características dos municípios do estado de Minas Gerais. Desenvolvimento em Questão, 10(20), 52-88. Recuperado de: https://doi. org/10.21527/2237-6453.2012.20.52-88

MENEZES, T.A. \& AZZONI, C.R. (2006). Convergência de salários entre as regiões metropolitanas brasileiras: custo de vida e aspectos de demanda e oferta de trabalho. Recuperado de: http://repositorio.ipea.gov.br/handle/11058/5054

PETINARI, R.A.; TERESO, M.J.A. \& BERGAMASCO, S.M.P.P. (2008). A importância da fruticultura para os agricultores familiares da região de]ales-SP. Revista Brasileira de Fruticultura, 30(2), 356-360. https://doi.org/10.1590/S0100-29452008000200015 RESENDE, M. (1994). Medidas de concentração industrial: uma resenha. Análise econômica, 12 (21 e 22). https://doi.org/10.22456/2176-5456.10488

RESENDE, M. \& BOFF, H. (2013). Concentração industrial. In Economia industrial, pp. 53-65. Elsevier Editora Ltda.

RODRIGUES, A.B.; MARTINS, M.I.E.G. \& ARAÚJO, J.D. (1997). Avaliação econômica da produção de alface em estufa. Informações econômicas, 27(1), 27-33. Recuperado de: http://www.iea.sp.gov.br/out/LerTexto.php?codTexto=997

SAMBUICHI, R.H.R.; GALINDO, E.P.; OLIVEIRA, M.Â.C.D.; \& PEREIRA, R.M. (2014). A diversificação produtiva como forma de viabilizar o desenvolvimento sustentável da agricultura familiar no Brasil. Em L. Monteiro Monasterio, M. Côrtes Neri \& S. Suarez Dillon Soares (Eds.), Brasil em desenvolvimento 2014. Estado, planejamento e políticas públicas (IPEA). Recuperado de: http://repositorio.ipea.gov.br/ handle/11058/3605

SÃOPAULO(ESTADO),SECRETARIADEAGRICULTURAEABASTECIMENTO.INSTITUTO DE ECONOMIA AGRÍCOLA. COORDENADORIA DE DESENVOLVIMENTO RURAL SUSTENTÁVEL (2009). Projeto LUPA (2007/08): Censo Agropecuário do Estado de São Paulo. São Paulo: SAA: IEA: CDRS. Recuperado de: http://www.cdrs.sp.gov.br/ projetolupa/index.php

SCHROTH, G. \& RUF, F. (2014). Farmer strategies for tree crop diversification in the humid tropics. A review. Agronomy for sustainable development, 34(1), 139-154. https://doi.org/10.1007/s13593-013-0175-4

SETIAWAN, M.; EMVALOMATIS, G. \& LANSINK, A.O. (2012). The relationship between technical efficiency and industrial concentration: Evidence from the Indonesian food and beverages industry. Journal of Asian Economics, 23(4), 466-475. https:// doi.org/10.1016/j.asieco.2012.01.002

SHANKAR, R. \& SHAH, A. (2003). Bridging the economic divide within countries: A scorecard on the performance of regional policies in reducing regional income disparities. World development, 31(8), 1421-1441. https://doi.org/10.1016/S0305750X(03)00098-6

SILVA, C.A.G. \& FERREIRA, L.D.R. (2016). Produtividade total dos fatores no crescimento da agropecuária brasileira. Revista de Política Agrícola, 25(3), 4-15. 
Recuperado de: https://seer.sede.embrapa.br/index.php/RPA/article/view/1151 SILVA, J.A. \& ANDRAZ, J.M. (2004). O padrão de especialização e a localização das actividades económicas no Algarve. Estudos I, 177-194. Recuperado de: http:// sapientia.ualg.pt/handle/10400.1/5151

SILVA, J.D.; COELHO, P.; CASER, D.; BUENO, C.; BINI, D.; PINATTI, E. \& CASTANHO FILHO, E.P. (2020). Valor da produção agropecuária do estado de São Paulo: resultado final 2019. Análises e indicadores. Recuperado de: http://www.iea.sp.gov. br/out/TerTexto.php?codTexto $=14778$

SILVA, J.G. \& GROSSI, E.D. (2001). Onovo rural brasileiro: uma atualização para 1992-98. Technical report, UNICAMP

SINGHA, A.K.; BARUAH, M.).; BORDOLOI, R.; DUTTA, P. \&SAIKIA, U.S. (2012). Analysis on influencing factors of technology adoption of different land based enterprises of farmers under diversified farming system. Journal of agricultural science, 4(2), 139. http://dx.doi.org/10.5539/jas.v4n2p139

SOUZA, P.M.; NEY, M.G.; FORNAZIER, A. \& PONCIANO, N.]. (2015). Comportamento da distribuição do valor da produção vegetal entre os estabelecimentos agropecuários: uma análise dos dados dos censos de 1995/96 e 2006. Revista Econômica do Nordeste, 46(1), 131-150. Recuperado de:https://www.bnb.gov.br/ revista/index.php/ren/article/view/184

VEIGA, J.E.D.; FAVARETO, A.; AZEVEDO, C.M.; BITTENCOURT, G.; VECCHIATTI, K. \& MAGALHÃES, R. (2001). O Brasil rural precisa de uma estratégia de desenvolvimento. Série textos para discussão, 1.

VILELA, N.J. \& MACEDO, M. (2000). Fluxo de poder no agronegócio: o caso das hortaliças. Horticultura Brasileira, 18(2), 88-94. https://doi.org/10.1590/S010205362000000200002

ZAMBOM, M.A.; SANTOS, G.T. DOS; MODESTO, E.C.; ALCALDE, C.R.; GONÇALVES, G.D.; SILVA, D.C. DA; SILVA, K.T. DA \& FAUSTINO, J.O. (2008). Valor nutricional da casca do grão de soja, farelo de soja, milho moído e farelo de trigo para bovinos. Acta Scientiarum. Animal Sciences, 23, 937-943. https://doi.org/10.4025/ actascianimsci.v23io.2648 